\title{
CHARACTERIZATIONS OF THE INVERSE \\ WEIBULL DISTRIBUTION AND GENERALIZED EXTREME VALUE DISTRIBUTIONS BY MOMENTS OF $k$ TH RECORD VALUES
}

Abstract. We give characterization conditions for the inverse Weibull distribution and generalized extreme value distributions by moments of $k$ th record values.

1. Introduction. We discuss characterization problems for an inverse Weibull distribution function

$$
F(x)=e^{-(\theta / x)^{\alpha}}, \quad x>0, \alpha>0, \theta>0,
$$

and the standard generalized extreme value distribution function given by

$$
F(x)= \begin{cases}e^{-\{1-\gamma x\}^{1 / \gamma},}, & x<1 / \gamma \text { when } \gamma>0, \\ & x>1 / \gamma \text { when } \gamma<0, \\ e^{-e^{-x}}, & x \in \mathbb{R} \text { when } \gamma=0 .\end{cases}
$$

Note that $F(x)$ given by (1.1) with $\theta=1$ is a Fréchet distribution function (cf. [3]).

We present characterization conditions for distribution functions given by (1.1) and (1.2) by moments of $k$ th lower record values introduced in [3]. The $k$ th upper record values were discussed in [1]. So first we recall the concept of $k$ th lower record values (cf. [3]).

Let $\left\{X_{n}, n \geq 1\right\}$ be a sequence of i.i.d. random variables with a cumulative distribution function $F(x)$ and a probability density function $f(x)$. The $j$ th order statistic of a sample $\left(X_{1}, \ldots, X_{n}\right)$ is denoted by $X_{j: n}$. For a fixed $k \geq 1$ we define the sequence $\left\{L_{k}(n), n \geq 1\right\}$ of $k$ th lower record times

2000 Mathematics Subject Classification: Primary 62E10, 60E99.

Key words and phrases: sample, order statistics, record values, $k$ th record values, inverse Weibull distribution, generalized extreme value distributions. 
of $\left\{X_{n}, n \geq 1\right\}$ as follows:

$$
\begin{aligned}
& L_{k}(1)=1, \\
& L_{k}(n+1)=\min \left\{j>L_{k}(n): X_{k: L_{k}(n)+k-1}>X_{k: j+k-1}\right\} .
\end{aligned}
$$

For $k=1$ we put $L(n):=L_{1}(n), n \geq 1$, which are lower record times of $\left\{X_{n}, n \geq 1\right\}$. The sequence $\left\{Z_{n}^{(k)}, n \geq 1\right\}$ with $Z_{n}^{(k)}=X_{k: L_{k}(n)+k-1}$, $n=1,2, \ldots$, is called the sequence of $k$ th lower record values of $\left\{X_{n}, n \geq 1\right\}$. For convenience, we also set $Z_{0}^{(k)}=0$. Note that for $k=1$ we have $Z_{n}^{(1)}=$ $X_{L(n)}, n \geq 1$, i.e. the record values of $\left\{X_{n}, n \geq 1\right\}$. Moreover, we see that $Z_{1}^{(k)}=\max \left(X_{1}, \ldots, X_{k}\right):=X_{k: k}$.

It is known (cf. [3]) that the pdf of $Z_{n}^{(k)}$ and the joint pdf of $\left(Z_{m}^{(k)}, Z_{n}^{(k)}\right)$ are given respectively by

$$
\begin{aligned}
& f_{Z_{n}^{(k)}}(x)=\frac{k^{n}}{(n-1) !}[-\ln F(x)]^{n-1}[F(x)]^{k-1} f(x), \quad n \geq 1, \\
& f_{Z_{m}^{(k)}, Z_{n}^{(k)}}(x, y)=\frac{k^{n}}{(m-1) !(n-m-1) !}[\ln F(x)-\ln F(y)]^{n-m-1} \\
& \times[-\ln F(x)]^{m-1} \frac{f(x)}{F(x)}[F(y)]^{k-1} f(y), \\
& x>y, 1 \leq m<n, n \geq 2 .
\end{aligned}
$$

Results for $k$ th upper record values can be found in [1].

Section 2 contains characterization conditions for an inverse Weibull distribution and in Section 3 we give recurrence relations for product moments of $k$ th lower record values of that distribution. Characterization conditions for the standard generalized extreme distribution (1.2) are presented in Section 4.

2. Characterization conditions for an inverse Weibull distribution. The characterizations of distributions presented in this paper are based on the following result by Lin (cf. [2]).

Proposition. Let $n_{0}$ be any fixed non-negative integer, $-\infty<a<b$ $<\infty$, and $g(x)>0$ an absolutely continuous function with $g^{\prime}(x) \neq 0$ a.e. on $(a, b)$. Then the sequence of functions $\left\{(g(x))^{n} e^{-g(x)}, n \geq n_{0}\right\}$ is complete in $L(a, b)$ iff $g(x)$ is strictly monotone on $(a, b)$.

Let us note that for the inverse Weibull distribution (1.1) we have

$$
x f(x)=\alpha F(x)(-\ln F(x)) .
$$

We start with recurrence relations for moments of the inverse Weibull distribution. From them we derive a formula for single moments expressed in terms of moments of $X_{k: k}$. 
TheOREM 1. Fix a positive integer $k \geq 1$. Then for any positive integer $r$, we have

$$
E\left(Z_{n}^{(k)}\right)^{r}=\left(1-\frac{r}{(n-1) \alpha}\right) E\left(Z_{n-1}^{(k)}\right)^{r}
$$

whenever $(n-1) \alpha>r$, and consequently,

$$
E\left(Z_{n}^{(k)}\right)^{r}=\prod_{i=1}^{n-1}\left(1-\frac{r}{i \alpha}\right) E\left(X_{k: k}\right)^{r} .
$$

Pr o of. For $n \geq 1$ and $r=1,2, \ldots$, from (1.3) we have

$$
E\left(Z_{n}^{(k)}\right)^{r}=\frac{\alpha k^{n}}{(n-1) !} \int x^{r-1}[-\ln F(x)]^{n}[F(x)]^{k} d x .
$$

Integrating by parts in the above integral written as $\int x^{r-1} d(\ldots)$, we get

$$
E\left(Z_{n-1}^{(k)}\right)^{r}=\frac{\alpha(n-1)}{r}\left[E\left(Z_{n-1}^{(k)}\right)^{r}-E\left(Z_{n}^{(k)}\right)^{r}\right] .
$$

This gives (2.2). Using an induction argument leads to (2.3).

COROLlary. Under the assumptions of Theorem 1 with $\alpha=1$ we obtain a recurrence relation for single moments of kth lower record values from the inverse exponential distribution:

$$
E\left(Z_{n}^{(k)}\right)^{r}=\left(1-\frac{r}{n-1}\right) E\left(Z_{n-1}^{(k)}\right)^{r} .
$$

Now we show that one can have a stronger result.

TheOREM 2. Fix a positive integer $k \geq 1$ and let $r$ be a positive integer. $A$ necessary and sufficient condition for a random variable $X$ to be distributed with pdf given by (1.1) is that

$$
E\left(Z_{n}^{(k)}\right)^{r}=\left(1-\frac{r}{(n-1) \alpha}\right) E\left(Z_{n-1}^{(k)}\right)^{r}
$$

for all positive integers $n$ such that $(n-1) \alpha>r$.

Proof. The necessity part follows immediately from Theorem 1.

On the other hand if the recurrence relation (2.2) is satisfied, then

$$
\int x^{r-1}[-\ln F(x)]^{n-1}[F(x)]^{k-1}\{x f(x)-\alpha(-\ln F(x)) F(x)\} d x=0 .
$$

It now follows from the Proposition that

$$
x f(x)=\alpha(-\ln F(x)) F(x),
$$

which proves by (2.1) that $f(x)$ has the form (1.1). 
Corollary. Let $\alpha=1$ in Theorem 2. A necessary and sufficient condition for a random variable $X$ to have the inverse exponential distribution is that

$$
E\left(Z_{n}^{(k)}\right)^{r}=\left(1-\frac{r}{n-1}\right) E\left(Z_{n-1}^{(k)}\right)^{r}
$$

for all positive integers $n$ such that $n-1>r$.

COROLlary. Under the assumptions of Theorem 2 with $r=1$ the equations

$$
E Z_{n}^{(k)}=\left(1-\frac{1}{(n-1) \alpha}\right) E Z_{n-1}^{(k)}, \quad(n-1) \alpha>1,
$$

characterize an inverse Weibull distribution.

ExAmple. Let $\prod_{j=1}^{0}(1-1 /(j \alpha)):=1$ and assume that $\alpha>1$. Then the equations

$$
E Z_{n}^{(k)}=\frac{\theta}{k^{\alpha}} \Gamma\left(1-\frac{1}{\alpha}\right) \prod_{j=1}^{n-1}\left(1-\frac{1}{j \alpha}\right) \quad \text { for } n=1,2, \ldots
$$

all hold iff

$$
F(x)=e^{-(\theta / x)^{\alpha}}, \quad x>0, \alpha>1 .
$$

REMARK. If we let additionally $k=1$ then

$$
E X_{L(n)}=\theta \Gamma\left(1-\frac{1}{\alpha}\right) \prod_{j=1}^{n-1}\left(1-\frac{1}{i \alpha}\right) \quad \text { for } n=1,2, \ldots
$$

iff $F(x)$ is given by (1.1) with $\alpha>1$.

Note that the assumption $\alpha>1$ is needed for the existence of $E X$.

3. Product moments of $k$ th lower record values from an inverse Weibull distribution. We complete our considerations by giving recurrence relations for product moments of $k$ th lower record values from an inverse Weibull distribution.

TheOREM 3. Fix a positive integer $k \geq 1$ and let $r$ be a non-negative integer such that $r<m \alpha$. Then for $m \geq 1, s=1,2, \ldots$,

$$
E\left(Z_{m+1}^{(k)}\right)^{r+s}=\left(1-\frac{r}{m \alpha}\right) E\left[\left(Z_{m}^{(k)}\right)^{r}\left(Z_{m+1}^{(k)}\right)^{s}\right]
$$

and for $1 \leq m \leq n-2$,

$$
E\left[\left(Z_{m+1}^{(k)}\right)^{r}\left(Z_{n}^{(k)}\right)^{s}\right]=\left(1-\frac{r}{m \alpha}\right) E\left[\left(Z_{m}^{(k)}\right)^{r}\left(Z_{n}^{(k)}\right)^{s}\right] .
$$


Pr o of. From (1.1) for $1 \leq m \leq n-1$ and $r, s=1,2, \ldots$,

$$
\begin{aligned}
E\left[\left(Z_{m}^{(k)}\right)^{r}\left(Z_{n}^{(k)}\right)^{s}\right] & =\iint x^{r} y^{s} f_{m, n}(x, y) d x d y \\
& =\frac{k^{n}}{(m-1) !(n-m-1) !} \int y^{s}[F(y)]^{k-1} f(y) I(y) d y
\end{aligned}
$$

where

$$
\begin{aligned}
I(y) & =\int x^{r}[-\ln F(x)]^{m-1} \frac{f(x)}{F(x)}[\ln F(x)-\ln F(y)]^{n-m-1} d x \\
& =\alpha \int x^{r-1}[-\ln F(x)]^{m}[\ln F(x)-\ln F(y)]^{n-m-1} d x .
\end{aligned}
$$

But

$$
\begin{aligned}
I(y) & =\frac{\alpha m}{r}\left[\int x^{r}[-\ln F(x)]^{m-1}[\ln F(x)-\ln F(y)]^{n-m-1} \frac{f(x)}{F(x)} d x\right] \\
& -\frac{\alpha(n-m-1)}{r}\left[\int x^{r}[-\ln F(x)]^{m}[\ln F(x)-\ln F(y)]^{n-m-2} \frac{f(x)}{F(x)} d x\right] .
\end{aligned}
$$

Upon substituting the above equation in (3.3) and simplifying, we obtain

$$
E\left[\left(Z_{m}^{(k)}\right)^{r}\left(Z_{n}^{(k)}\right)^{s}\right]=\frac{\alpha m}{r}\left[E\left[\left(Z_{m}^{(k)}\right)^{r}\left(Z_{n}^{(k)}\right)^{s}\right]-E\left[\left(Z_{m+1}^{(k)}\right)^{r}\left(Z_{n}^{(k)}\right)^{s}\right]\right]
$$

Hence we have (3.2). When $n=m+1$ we obtain (3.1).

4. Characterization conditions for the generalized extreme value distribution. Recurrence relations for moments of $k$ th lower record values from a generalized extreme value distribution were presented in [3]. We now only give characterization conditions for the standard generalized extreme value distribution (1.2) based on those relations.

To characterize the df $F$ given by (1.2) we use an equivalent representation of (1.2), namely

$$
(1-\gamma x) f(x)=F(x)(-\ln F(x)) \quad \text { for } \gamma \neq 0,
$$

and $f(x)=F(x)(-\ln F(x))$ for $\gamma=0$.

The main result of this section is as follows.

THEOREM 4. A necessary and sufficient condition for a random variable $X$ to be distributed according to (2.1) is that

$$
E\left(Z_{n}^{(k)}\right)^{r}=\left(1+\gamma \frac{r}{n-1}\right) E\left(Z_{n-1}^{(k)}\right)^{r}-\frac{r}{n-1}\left(E Z_{n-1}^{(k)}\right)^{r-1}
$$

for $n=2,3, \ldots$

Proof. The necessity part was proved in [3]. Assume now that (4.2) is satisfied. Then

$$
\int[-\ln F(x)]^{n-1}[F(x)]^{k-1} x^{r}[-(-\ln F(x)) F(x)-\gamma x f(x)+f(x)] d x=0 .
$$


It now follows from the above Proposition that

$$
(1-\gamma x) f(x)=(-\ln F(x)) F(x),
$$

which proves that

$$
f(x)=e^{(1-\gamma x)^{1 / \gamma}}(1-\gamma x)^{1 / \gamma-1} .
$$

EXAmPLE. Let $\prod_{j=n}^{n-1}(1+\gamma / j):=1$. Then for $\gamma>0$,

$$
\begin{aligned}
E Z_{n}^{(k)}= & \frac{1}{\gamma}\left(1-\frac{1}{k^{\gamma}} \Gamma(\gamma+1)\right) \prod_{j=1}^{n-1}\left(1+\frac{\gamma}{j}\right) \\
& -\sum_{i=1}^{n-1} \frac{1}{i} \prod_{j=i+1}^{n-1}\left(1+\frac{\gamma}{j}\right) \quad \text { for } n=2,3, \ldots
\end{aligned}
$$

iff $F(x)$ is given by (1.2).

REMARK. If we let additionally $k=1$, then

$$
\begin{aligned}
X_{L(n)}= & \frac{1}{\gamma}(1-\Gamma(\gamma+1)) \prod_{j=1}^{n-1}\left(1+\frac{\gamma}{j}\right) \\
& -\sum_{i=1}^{n-1} \frac{1}{i} \prod_{j=i+1}^{n-1}\left(1+\frac{\gamma}{j}\right) \quad \text { for } n=2,3, \ldots
\end{aligned}
$$

iff $F(x)$ is given by (1.2).

Acknowledgments. We are grateful to the referee for his comments which allowed us to give a more general presentation of the results.

\section{References}

[1] W. Dziubdziela and B. Kopociński, Limiting properties of the $k$-th record value, Zastos. Mat. 15 (1976), 187-190.

[2] G. D. Lin, On a moment problem, Tôhoku Math. J. 38 (1986), 595-598.

[3] P. Pawlas and D. Szynal, Relations for single and product moments of $k$-th record values from exponential and Gumbel distribution, J. Appl. Statist. Sci. 7 (1998), $53-62$.

Piotr Pawlas and Dominik Szynal Department of Mathematics

Maria Curie-Skłodowska University

Pl. M. Curie-Skłodowskiej 1

20-031 Lublin, Poland

E-mail: szynal@golem.umcs.lublin.pl 